\title{
Element-Specific Probing of Ultrafast Spin Dynamics in Multisublattice Magnets with Visible Light
}

\author{
A. R. Khorsand, ${ }^{1, *}$ M. Savoini, ${ }^{1}$ A. Kirilyuk, ${ }^{1}$ A. V. Kimel, ${ }^{1}$ A. Tsukamoto, ${ }^{2,3}$ A. Itoh, ${ }^{2}$ and Th. Rasing ${ }^{1}$ \\ ${ }^{1}$ Institute for Molecules and Materials, Radboud University Nijmegen, Heyendaalseweg 135, 6525 AJ Nijmegen, The Netherlands \\ ${ }^{2}$ College of Science and Technology, Nihon University, 7-24-1 Funabashi, Chiba, Japan \\ ${ }^{3}$ Japan Science and Technology Agency, PRESTO, 4-1-8 Honcho Kawaguchi, Saitama, Japan
}

(Received 5 July 2012; published 7 March 2013)

\begin{abstract}
We demonstrate the feasibility of element-specific probing of ultrafast spin dynamics in the multisublattice magnet $\mathrm{TbFe}$ in the visible spectral range. In particular, we show that one can selectively study the dynamics of $\mathrm{Tb}$ and $\mathrm{Fe}$ sublattices choosing the wavelength of light below and above $610 \mathrm{~nm}$, respectively. We observe that, despite their antiferromagnetic coupling in the ground state, the $\mathrm{Tb}$ and $\mathrm{Fe}$ spins temporarily align ferromagnetically after excitation with an intense 55-fs laser pulse, after which they relax to their initial states due to the strong anisotropy in $\mathrm{Tb}$.
\end{abstract}

PACS numbers: 75.50.Gg, 42.62.Fi, 71.70.Gm, 75.50.Ss

Femtosecond laser excitation of multisublattice magnets opens the way to trigger ultrafast spin dynamics and even magnetization reversal driven by the strong exchange interaction between the sublattices $[1,2]$. To explore and exploit these intriguing possibilities requires femtosecond time-resolved and element-specific studies of the spin dynamics in multisublattice systems.

The wave functions of the magnetic states in metals probed in the visible spectral range have, in general, a substantial overlap. This fact has been a serious obstacle for experimental studies of element-specific ultrafast magnetization dynamics. Until now it has been a conventionally accepted wisdom that ultrafast and element-specific probing of magnetism in the visible spectral range is hardly possible, and only photons in the soft-x-ray $[2,3]$ and EUV [4] regimes were used for such studies probing transitions of strongly localized electrons whose exchange split energy levels serve as the fingerprint of the element.

However, techniques utilizing high-energy photons have some great limitations, in particular, in combination with subpicosecond time resolution. For instance, the number of photons per pulse is small and subject to fluctuations, giving rise to an extremely low signal-to-noise ratio and time-consuming measurements. Furthermore, the generation of soft-x-ray fs pulses requires rather unique and demanding sources, and the access to such facilities is very limited.

In this Letter, we formulate requirements which allow element-specific probing in the visible spectral range. We study ultrafast laser-induced magnetization dynamics of a $\mathrm{TbFe}$ alloy, and find that one can selectively study the

Published by the American Physical Society under the terms of the Creative Commons Attribution 3.0 License. Further distribution of this work must maintain attribution to the author(s) and the published article's title, journal citation, and DOI. dynamics of $\mathrm{Tb}$ and $\mathrm{Fe}$ spins choosing the wavelength of light below and above $610 \mathrm{~nm}$, respectively. Furthermore, we find that at high excitation intensities the antiferromagnetically coupled spins temporarily align ferromagnetically, succeeded with magnetization recovery, in striking contrast to $\mathrm{GdFe}$ where the magnetization reverses.

The feasibility of element-specific spin dynamics with visible light is investigated on a TbFe-based alloy. The magnetic material is an amorphous, thin ferrimagnetic film of $20 \mathrm{~nm}$. The composition is $16 \% \mathrm{~Tb}, 75 \% \mathrm{Fe}$, and $9 \% \mathrm{Co}$, with an out-of-plane anisotropy and a coercive field of $1 \mathrm{~T}$. The magnetic moments of $\mathrm{Tb}$ and $\mathrm{FeCo}$ form two antiferromagnetically coupled sublattices with similar, though nonequivalent, magnetic moments at room temperature. In the following, we focus on the behavior of $\mathrm{Tb}$ and $\mathrm{Fe}$ and ignore the small contribution of angular momentum of Co.

Both static magneto-optic Kerr effect (MOKE) and time-resolved (TR) MOKE measurements [5-9] were performed at room temperature. We define the Kerr rotation as the difference in rotation of linearly polarized light for positive and negative magnetization, i.e., $\theta_{K}=\theta_{+M^{-}} \theta_{-M}$. This ensures that $\theta_{K}$ is of a purely magnetic origin. In Fig. 1(a) the spectral dependence of the static Kerr rotation $\theta_{K, 0}$ is shown, i.e., the Kerr rotation of $\mathrm{TbFe}$ in the absence of an excitation pulse. The spectrum contains three features: (i) a small but positive signal in the (infra)red regime, (ii) a sign change around $610 \mathrm{~nm}$, and (iii) a large and relatively sharp peak around $500 \mathrm{~nm}$.

In a material with two magnetic sublattices, the Kerr rotation consists of the collective response of both magnetic species. In an isotropic medium in a polar geometry [see inset of Fig. 2(b)], the Kerr rotation is in first order approximation given by $[10,11]$

$$
\theta_{K}(\lambda)=\theta_{1}(\lambda)+\theta_{2}(\lambda)=K_{1}(\lambda) M_{1}+K_{2}(\lambda) M_{2},
$$

where $\theta_{i}, M_{i}$, and $K_{i}$ are the Kerr rotation, out-of-plane component of the magnetization, and MO susceptibility of 


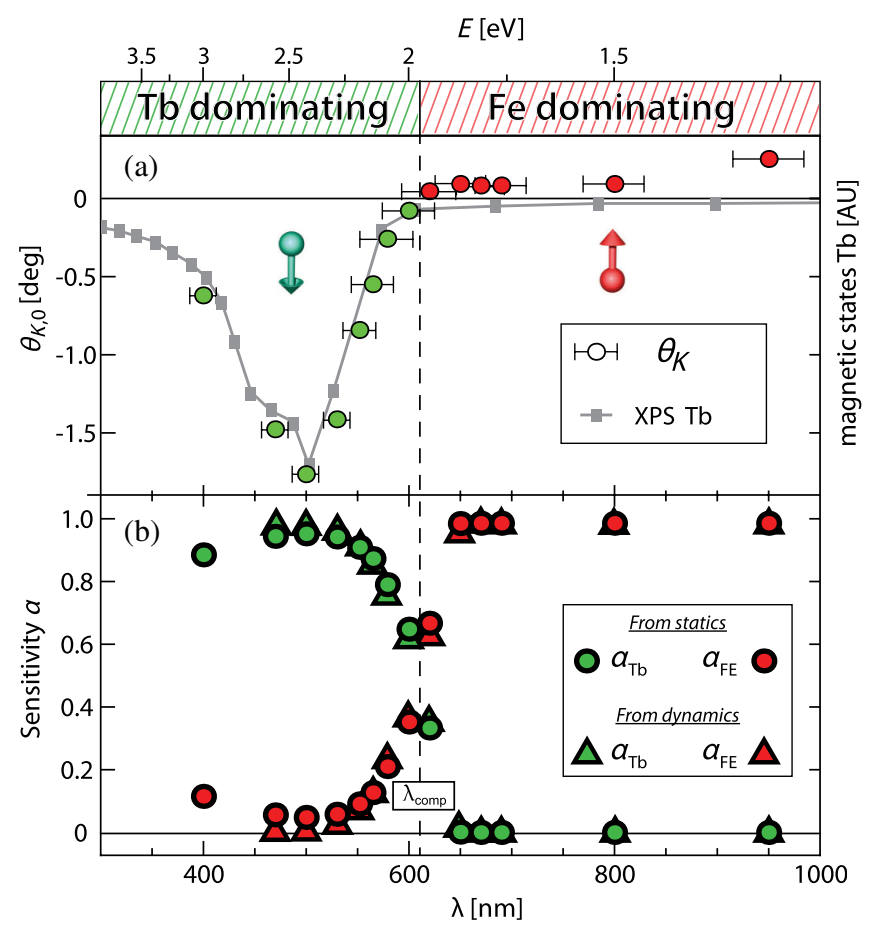

FIG. 1 (color online). Response of the TbFe alloy as a function of the probe wavelength (bottom axis) and photon energy (top axis). (a) The static Kerr rotation (dots), and the magnetic states in the $4 f$ shell of $\mathrm{Tb}$ (solid line). The latter was measured with spin-polarized x-ray photoemission (adapted from Ref. [12]). (b) The sensitivity $\alpha$ to $\mathrm{Fe}$ (red) and $\mathrm{Tb}$ (green) sublattices obtained from static MOKE (dots) and TR MOKE (triangles) measurements.

sublattice $i$, respectively. The probe sensitivity $\alpha_{i}$ to sublattice $i$ is defined with

$$
\alpha_{i}(\lambda)=\frac{\left|\theta_{i}(\lambda)\right|}{\left|\theta_{\mathrm{Tb}}(\lambda)\right|+\left|\theta_{\mathrm{Fe}}(\lambda)\right|} .
$$

$K_{\mathrm{Fe}}$ and $K_{\mathrm{Tb}}$ have the same sign and do not reverse at $\lambda=400-950 \mathrm{~nm}[10,11]$, whereas $M_{\mathrm{Fe}}$ and $M_{\mathrm{Tb}}$ have opposite signs due to the antiferromagnetic coupling. Therefore, the Kerr rotations of the $\mathrm{Tb}$ and Fe sublattices are opposite to each other. When their magnitudes are equivalent, i.e., $\alpha_{\mathrm{Fe}}=\alpha_{\mathrm{Tb}}=0.5$, the net Kerr rotation of $\mathrm{TbFe}$ goes to zero. This is the case around $610 \mathrm{~nm}$. Above and below this wavelength, the sign of the net Kerr rotation is given by the sublattice with the dominant contribution to the MO contrast; i.e., the sublattice for which $\alpha_{i}>0.5$. We identify the wavelength regimes above and below $610 \mathrm{~nm}$ as the $\mathrm{Fe}$ and $\mathrm{Tb}$ dominated regimes, respectively.

In magnetic transition metals (TMs) (e.g., $\mathrm{Fe}, \mathrm{Ni}$, and Co), the magnetic moment is carried by itinerant $3 d$ electrons near the Fermi level $\left(\mathrm{E}_{\mathrm{F}}\right)$. Because of the many possible low-energy transitions within the conduction band of these ferromagnets, their Kerr rotations are significant in the infrared (IR) regime. On the other hand, the largest part of the magnetic moment of rare-earth metals is

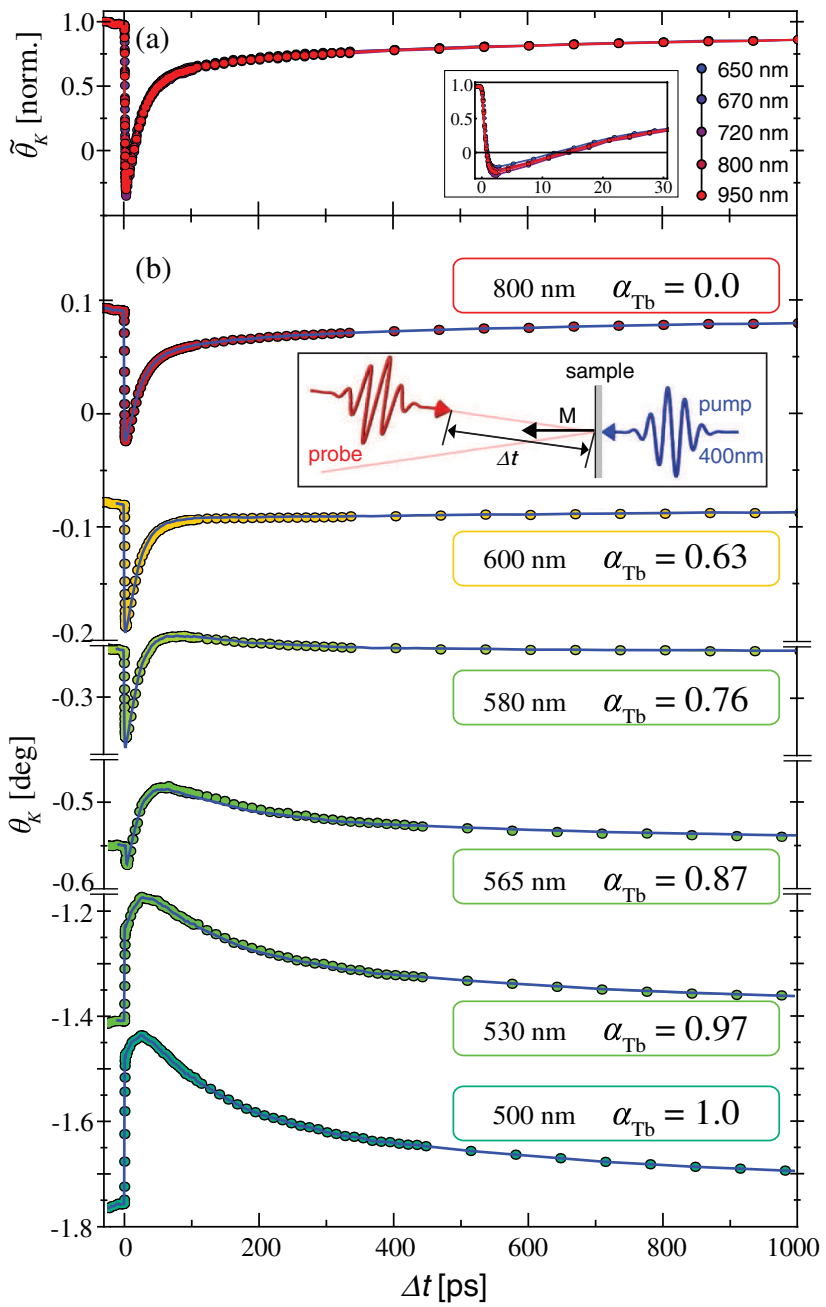

FIG. 2 (color online). Spectral dependence of TR MOKE measurements at a fixed excitation fluence of $4.4 \mathrm{~mJ} / \mathrm{cm}^{2}$. TR MOKE at (a) $\lambda=650-950 \mathrm{~nm}$ and (b) $\lambda=500-800 \mathrm{~nm}$. The blue solid lines in (b) are the fits to Eq. (3), where the fitting parameters $\alpha_{\mathrm{Tb}}$ are given at the right at each wavelength. Inset: Geometry of the pump-probe pulses with respect to the sample.

carried by the localized $4 f$ electrons [12-14], which is roughly $97 \%$ in $\mathrm{Tb}$. The other $3 \%$ is carried by $5 d$ conduction electrons. Spin-polarized XPS data of Tb are shown in Fig. 1(a) (adapted from Ref. [12]), from which we find that the $4 f$ shell is around $2.4 \mathrm{eV}$ below $\mathrm{E}_{\mathrm{F}}$. The $4 f$ electrons of $\mathrm{Tb}$ become accessible in the visible spectral range, and also the conduction electrons are accessible at $2-3 \mathrm{eV}$ [15]. This gives rise to a high Kerr rotation of the $\mathrm{Tb}$ sublattice in this regime. In fact, it can be seen from the XPS data in Fig. 1(a) that the large peak of the Kerr rotation around $500 \mathrm{~nm}$ is very closely related to the spinpolarized states of $\mathrm{Tb}$, indicating that the MO response comes mainly from $\mathrm{Tb}$.

The wavelength dependence of the MO response is studied in more detail with TR MOKE measurements at a fixed excitation fluence of $4.4 \mathrm{~mJ} / \mathrm{cm}^{2}$. First, we consider the dynamics in the regime $\lambda \geq 650 \mathrm{~nm}$ shown in 
Fig. 2(a). The normalized transient Kerr rotation, i.e., $\tilde{\theta}_{K}(\lambda, t) \equiv \frac{\theta_{K}(\lambda, t)}{\theta_{K, 0}(\lambda)}$, is plotted as a function of the pumpprobe delay time for various probe wavelengths. It can be seen that the MO response is independent of the probe wavelength, which demonstrates that the sensitivities $\alpha_{\mathrm{Fe}}$ and $\alpha_{\mathrm{Tb}}$ are constant at $\lambda>650 \mathrm{~nm}$.

At shorter wavelengths, the probe is sensitive to more than one sublattice. Otherwise, the magnetization dynamics $M_{i}(t) / M_{0}$ of sublattice $i$ would be given by $\theta_{K}(t) / \theta_{K, 0}$, and the measurements at 565-600 nm would have the unphysical implication that the absolute value of the magnetic moment increases shortly after excitation. On the other hand, if the measurements give the collective response of two sublattices, it should be possible to reproduce all profiles from two traces, i.e., from the $\mathrm{Tb}$ and $\mathrm{Fe}$ dominated responses at 500 and $800 \mathrm{~nm}$, respectively. We can examine this by fitting the dynamics measurements to the following function:

$\theta_{\mathrm{fit}}\left(\lambda, t, \alpha_{1}\right)=\theta_{K, 0}(\lambda) \frac{\left(1-\alpha_{\mathrm{Tb}}\right) \tilde{\theta}_{\mathrm{Fe}}(t)-\alpha_{\mathrm{Tb}} \tilde{\theta}_{\mathrm{Tb}}(t)}{1-2 \alpha_{\mathrm{Tb}}}$,

where $\theta_{K, 0}(\lambda)$ is the static Kerr rotation taken from Fig. 1(a), and $\tilde{\theta}_{\mathrm{Tb}}(t)$ and $\tilde{\theta}_{\mathrm{Fe}}(t)$ are the normalized time resolved Kerr rotations at 500 and $800 \mathrm{~nm}$, respectively. The denominator $\left(1-2 \alpha_{\mathrm{Tb}}\right)$ ensures that the right-hand term is normalized to +1 at $t<0$. We note that we use only one fitting parameter, namely, $\alpha_{\mathrm{Tb}}$, in order to fit the whole set of time dependencies at probe wavelength $\lambda$. The fits are shown in Fig. 2(b) as solid blue lines and are in excellent agreement with the measurements. This demonstrates that there are only two traces that give rise to the TR MOKE measurements at $\lambda=500-950 \mathrm{~nm}$, and validates Eq. (1) in this wavelength range.

Having demonstrated that we can readily measure different parts of the magnetization of $\mathrm{TbFe}$ by changing the probe wavelength, we have a closer look at the MO responses at 500 and $800 \mathrm{~nm}$. The demagnetization profiles show features which are typical for $\mathrm{Fe}$ and $\mathrm{Tb}$. The demagnetization at $800 \mathrm{~nm}$ is ultrafast $(\tau \approx 0.2 \mathrm{ps})$, succeeded by a recovery. Such "one-step demagnetization" is typical for $3 d$ TMs like $\mathrm{Fe}$ [5-8]. On the other hand, at $500 \mathrm{~nm}$ the drop of the MO signal is initially ultrafast $(\tau \approx 0.4 \mathrm{ps})$, succeeded by slow demagnetization $(\tau \approx 15 \mathrm{ps})$ before it recovers. Such "two-step demagnetization" is typical for rare-earth metals such as $\mathrm{Tb}[7,16]$, and is generally assigned to their large magnetic moments. Therefore, the TR MOKE measurements at 500 and $800 \mathrm{~nm}$ reveal fingerprints of the $\mathrm{Tb}$ and Fe sublattices, respectively. This is in agreement with the static MOKE measurements shown in Fig. 1(a) from which we concluded that the probe is sensitive to dominantly the $\mathrm{Tb}$ and Fe sublattice at wavelengths below and above $610 \mathrm{~nm}$, respectively.

Apart from a different shape of the demagnetization profile, there is a large difference in the change in the MO contrast. The maximum change of the MO contrast

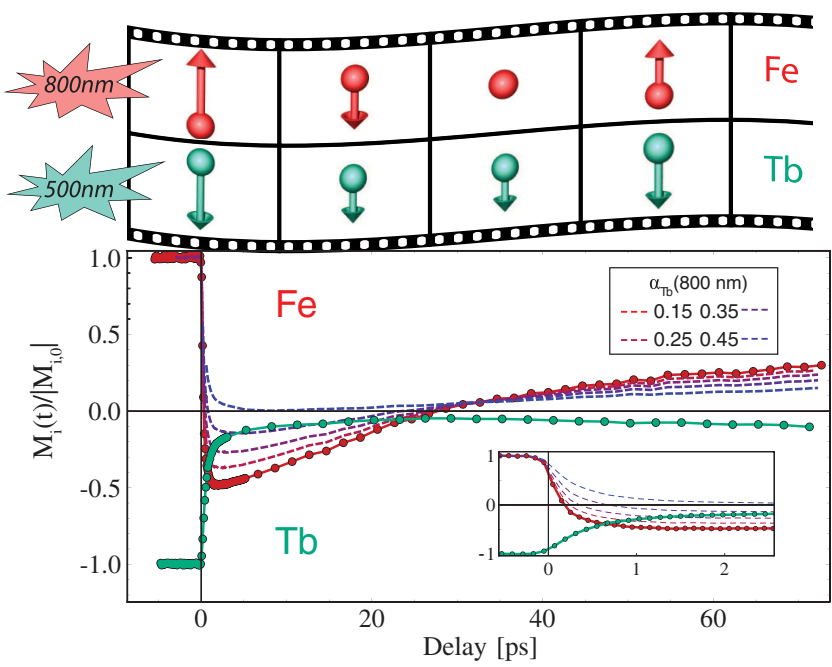

FIG. 3 (color online). Ultrafast sublattice magnetization dynamics of TbFe at $\lambda_{\mathrm{Fe}}=800 \mathrm{~nm}$ and $\lambda_{\mathrm{Tb}}=500$, corresponding to dominantly the $\mathrm{Fe}$ and $\mathrm{Tb}$ sublattices, respectively. The excitation fluence was $8 \mathrm{~mJ} / \mathrm{cm}^{2}$. The dashed lines give the magnetization dynamics of $\mathrm{Fe}$ for various sensitivities to $\mathrm{Tb}$ at $800 \mathrm{~nm}$.

is much smaller at $500 \mathrm{~nm}(\approx 20 \%)$ compared to $800 \mathrm{~nm}$ $(>100 \%)$. At an excitation intensity of $8 \mathrm{~mJ} / \mathrm{cm}^{2}$ shown in Fig. 3, these values are $95 \%$ and $150 \%$, respectively.

The magnetization dynamics of each sublattice can be extracted from the TR MOKE measurements once the probe sensitivity to each sublattice is known at 500 and $800 \mathrm{~nm}$. In general, the sublattice sensitivities at different optical wavelengths could be deduced with a calibration from x-ray magnetic circular dichroism (XMCD) measurements. This can be done by fitting one series of TR XMCD measurements at the $\mathrm{Fe}$ and $\mathrm{Tb}$ edges to TR MOKE traces at $\lambda_{1}$ and $\lambda_{2}$ measured under the same conditions, with a similar fitting as with Eq. (3). In fact, when TR MOKE measurements are combined with such calibration measurements, element-specific information could be obtained with visible light for two-sublattice magnets in general. The only condition is that the probe sensitivity to the two sublattices is different at $\lambda_{1}$ and $\lambda_{2}$ [17]. This condition is met in many alloys, such as in rare-earth-metal-transitionmetal alloys but also in TM-TM alloys such as FeNi. For example, $\theta_{K}$ of $\mathrm{Fe}$ and $\mathrm{Ni}$ have very different spectral dependencies: at $300 \mathrm{~nm} \theta_{K, \mathrm{Ni}} \approx 0^{\circ}$ and $\theta_{K, \mathrm{Fe}} \approx 0.3^{\circ}$, whereas at $400 \mathrm{~nm} \theta_{K, \mathrm{Ni}} \approx 0.2^{\circ}$, and $\theta_{K, \mathrm{Fe}} \approx 0.3^{\circ}$ [18]. Once the probe sensitivities are extracted from the calibration measurements, sublattice dynamics can be studied with visible light at different conditions, such as different excitation intensities or magnetic fields. Thus, despite the calibration with high-energy photons, this procedure allows element-specific measurements with the unique advantages of an optical probe.

For $\mathrm{TbFe}$, however, we can estimate the probe sensitivity to the $\mathrm{Fe}$ and $\mathrm{Tb}$ sublattices as their magnetization is 
largely contained at different energy levels. We use the static MOKE and TR MOKE measurements, in combination with the fact that the Kerr rotation of pure Fe decreases only a little in the regime $\lambda<650 \mathrm{~nm}$ [18]. Using $\theta_{\mathrm{Fe}}(\lambda) \lesssim \approx 90 \mathrm{mdeg}$, the sensitivity to the Tb sublattice at $\lambda=400-650 \mathrm{~nm}$ can be readily calculated with Eq. (2) and is shown in Fig. 1(b). We find that at $500 \mathrm{~nm}$ almost all signal comes from $\mathrm{Tb}$, i.e., $\alpha_{\mathrm{Tb}}>0.95$.

On the other hand, TR MOKE measurements have revealed that at longer wavelengths $(650-950 \mathrm{~nm})$ the sensitivities $\alpha_{\mathrm{Tb}}$ and $\alpha_{\mathrm{Fe}}$ are constant. In this low-energy regime, MO properties arise from transitions within the conduction band. Transition metals such as Fe have a relatively large MO susceptibility in this regime as their magnetic moments are contained within the conduction band. The $\mathrm{Tb}$ sublattice can also contribute to the Kerr rotation in the IR regime through the $5 d$ conduction electrons which carry approximately $3 \%$ of the magnetic moment. The Kerr rotation of $\mathrm{Tb}$, however, is typically very small compared to $\mathrm{Fe}$. We measured the Kerr rotations of $20 \mathrm{~nm}$ thin $\mathrm{Tb}$ and $\mathrm{Fe}$ films at $800 \mathrm{~nm}$ to be $\theta_{\mathrm{Tb}} \approx 10 \mathrm{mdeg}$ (at $100 \mathrm{~K}$ ) and $\theta_{\mathrm{Fe}} \approx$ 350 mdeg, respectively. We estimate from this that the contribution of $\mathrm{Tb}$ to the $\mathrm{MO}$ signal of $\mathrm{TbFe}$ is negligible in the IR regime and that $\alpha_{\mathrm{Fe}} \approx 1$. The thus obtained values are shown in Fig. 1(b) and are in excellent agreement with the fitted values from the dynamics measurements in Fig. 2.

It should be noted that the relative Kerr rotations of $\mathrm{Tb}$ and $\mathrm{Fe}$ in alloys may deviate from their pure forms. Using Eq. (3), we calculated the sublattice magnetization dynamics of Fe from $\theta_{K}(t, 800 \mathrm{~nm})$ for $0<\alpha_{\mathrm{Tb}}<0.5$. It can be seen that even under the conservative assumption that $\alpha_{\mathrm{Fe}}=0.65\left(\alpha_{\mathrm{Tb}}=0.35\right)$, the TR MOKE measurement at $800 \mathrm{~nm}$ indicates that the magnetization of Fe temporarily reverses sign. Only in the coincidental case that the relative contribution of $\mathrm{Tb}$ and $\mathrm{Fe}$ is almost equal at $800 \mathrm{~nm}$, i.e., $1<\left|\theta_{\mathrm{Fe}} / \theta_{\mathrm{Tb}}\right|<1.2\left(0.45<\alpha_{\mathrm{Tb}}<0.5\right)$, then the magnetization of the Fe sublattice would not reverse. We find this very unlikely as this ratio is much smaller compared to their pure forms, i.e., $\left|\theta_{\mathrm{Fe}} / \theta_{\mathrm{Tb}}\right| \approx 35$. Furthermore, a net Kerr rotation of $\approx 300$ mdeg at $950 \mathrm{~nm}$ with $\alpha_{\mathrm{Tb}}>0.45$ would imply that the absolute Kerr rotations of the $\mathrm{Tb}$ and Fe sublattices would be larger than 1.35 and $1.65 \mathrm{deg}$, respectively. Thus, correcting for the small fraction of $\mathrm{Tb}$ in $\mathrm{TbFe}$, this would give the improbable implication that the Kerr rotation of $\mathrm{Tb}$ would be 3 orders of magnitude larger in this alloy compared to pure $\mathrm{Tb}$.

Therefore, the reversal of the MO contrast at $800 \mathrm{~nm}$ is assigned to the reversal of the $\mathrm{Fe}$ magnetization. This reversal leads to a transient magnetic state in which both sublattices are aligned ferromagnetically, despite their antiferromagnetic coupling in the ground state. A transient ferromagnetic state was recently observed in GdFeCo [2], which has an antiferromagnetic coupling in its ground state, too. It was demonstrated that such a transient ferromagnetic magnetic state is a necessary and sufficient condition for all-optical magnetization reversal in $\mathrm{GdFeCo}$ $[2,19]$; i.e., the reversal of both magnetic sublattices with a single laser pulse $[1,20]$. In $\mathrm{TbFeCo}$, however, we observe a different behavior; the transient ferromagnetic state is succeeded by the recovery of the magnetization.

The striking difference in the dynamics of $\mathrm{TbFeCo}$ and GdFeCo may be explained by the large difference in spin-orbit coupling in the two rare-earth-metal materials. Gadolinium has an exactly half-filled $4 f$ shell. Therefore, its net orbital moment is zero and the spinorbit coupling is very weak. As a consequence, the spins of the Gd atoms are coupled only weakly to the lattice, allowing them to follow the Fe spins relatively easily. Terbium, on the other hand, is known for its strong spinorbit coupling. Therefore, Tb spins are not only coupled to the spins of the $\mathrm{Fe}$ sublattice, but also to the lattice. This leads to two competing forces acting on the $\mathrm{Tb}$ spins. So far, multisublattice dynamics is described with a Hamiltonian which takes into account the spin-spin interactions, and neglects the anisotropy due to the spinorbit coupling [19]. This approximation is valid for GdTM alloys due to the small anisotropy in Gd. However, for an adequate description of the dynamics of other $4 f$ rare-earth metals such as $\mathrm{Tb}$, the anisotropy can not be ignored and should be included in the Hamiltonian.

Finally, element-specific probing with visible light has unique advantages compared to techniques utilizing highenergy photons [2-4], such as (i) superior signal-to-noise ratio due to the high photon flux, (ii) much less timeconsuming and demanding measurements, (iii) no need for a vacuum environment, (iv) no need to work with unique sources with limited access or buy expensive tools for the generation of high-energy photons, and (v) the use of nonionizing photons. Unique advantages of XMCD measurements are the possibilities to resolve the spin and orbital angular moments [21] and to have a spatial resolution of only several $\mathrm{nm}$.

In summary, we have demonstrated the feasibility of element-specific probing of ultrafast spin dynamics with (near-)visible light in a TbFe alloy. The antiferromagnetically coupled spins are brought in a transient ferromagnetic state at high excitation intensities succeeded with magnetization recovery. This is in striking contrast to GdFe, where a transient ferromagnetic state is succeeded with magnetization reversal. Furthermore, element-specific probing with visible light can be generalized to other multisublattice alloys when a single calibration with TR $\mathrm{XMCD}$ measurements is made.

We thank A. van Roij and A. Toonen for technical support. This research has received funding from Stichting voor Fundamenteel Onderzoek der Materie (FOM), De Nederlandse Organisatie voor Wetenschappelijk Onderzoek (NWO), and EC FP7 ITN Grant No. 214810 (FANTOMAS) and ERC Grant No. 257280 (Femtomagnetism). 
*s.khorsand@science.ru.nl

[1] C. D. Stanciu, F. Hansteen, A. V. Kimel, A. Kirilyuk, A. Tsukamoto, A. Itoh, and T. Rasing, Phys. Rev. Lett. 99, 047601 (2007).

[2] I. Radu et al., Nature (London) 472, 205 (2011).

[3] C. Stamm et al., Nat. Mater. 6, 740 (2007).

[4] C. La-O-Vorakiat, M. Siemens, M. M. Murnane, H. C. Kapteyn, P. Grychtol, R. Adam, C. M. Schneider, J. M. Shaw, H. Nembach, and T. J. Silva, Phys. Rev. Lett. 103, 257402 (2009).

[5] E. Beaurepaire, J. C. Merle, A. Daunois, and J. Y. Bigot, Phys. Rev. Lett. 76, 4250 (1996).

[6] B. Koopmans, M. van Kampen, J. T. Kohlhepp, and W. J. M. de Jonge, Phys. Rev. Lett. 85, 844 (2000).

[7] B. Koopmans, G. Malinowski, F. D. Longa, D. Steiauf, M. Fähnle, T. Roth, M. Cinchetti, and M. Aeschlimann, Nat. Mater. 9, 259 (2010).

[8] L. Guidoni, E. Beaurepaire, and J. Y. Bigot, Phys. Rev. Lett. 89, 017401 (2002).

[9] M. Sultan, U. Atxitia, A. Melnikov, O. ChubykaloFesenko, and U. Bovensiepen, Phys. Rev. B 85, 184407 (2012).

[10] P. Hansen, Handbook of Magnetic Materials, edited by K. Buschow (Elsevier, New York, 1991), Vol. 6.

[11] P. Hansen, C. Clausen, G. Much, M. Rosenkranz, and K. Witter, Jpn. J. Appl. Phys. 66, 756 (1989).

[12] E. Arenholz, E. Navas, K. Starke, L. Baumgarten, and G. Kaindl, Phys. Rev. B 51, 8211 (1995).
[13] S. Hufner, F. Schumann, E. Rotenberg, J. Tobin, S. H. Yang, B. S. Mun, S. Morton, J. Schafer, and D. Ehm, Phys. Rev. B 63, 085106 (2001).

[14] J. K. Lang, Y. Baer, and P. A. Cox, J. Phys. F 11, 121 (1981).

[15] K. M. Dobrich, G. Bihlmayer, K. Starke, J. E. Prieto, K. Rossnagel, H. Koh, E. Rotenberg, S. Blugel, and G. Kaindl, Phys. Rev. B 76, 035123 (2007).

[16] M. Wietstruk, A. Melnikov, C. Stamm, T. Kachel, N. Pontius, M. Sultan, C. Gahl, M. Weinelt, H. A. Dürr, and U. Bovensiepen, Phys. Rev. Lett. 106, 127401 (2011).

[17] See Supplemental Material at http://link.aps.org/ supplemental/10.1103/PhysRevLett.110.107205 for a discussion on the feasibility of element-specific probing with visible light in any two-sublattice magnets employing a calibration of probe sensitivities with TR XMCD measurements.

[18] A. Delin, O. Eriksson, B. Johansson, S. Auluck, and J. M. Wills, Phys. Rev. B 60, 14105 (1999).

[19] J. H. Mentink, J. Hellsvik, D. V. Afanasiev, B. A. Ivanov, A. Kirilyuk, A. V. Kimel, O. Eriksson, M. I. Katsnelson, and T. Rasing, Phys. Rev. Lett. 108, 057202 (2012); T. A. Ostler et al., Nat. Commun. 3, 666 (2012).

[20] A. R. Khorsand, M. Savoini, A. Kirilyuk, A. V. Kimel, A. Tsukamoto, A. Itoh, and T. Rasing, Phys. Rev. Lett. 108, 127205 (2012).

[21] B. T. Thole, P. Carra, F. Sette, and G. van der Laan, Phys. Rev. Lett. 68, 1943 (1992). 